2011-2021 : crises, ruptures et nouvelles dynamiques.

Dix ans après la triple catastrophe du 11 mars

\title{
Kamaishi face aux crises pluridimensionnelles d'après-guerre. Catastrophes, restructuration industrielle et décroissance démographique
} 戦後釜石における危機の多層化:次害・座業構造転換・人口収縮 Kamaishi in the Face of Post-War Multidimensional Crises. Disasters, Industrial Reconstruction and Population Decline

\section{Naofumi Nakamura}

Traducteur : Amira Zegrour

\section{CpenEdition}

Édition électronique

URL : https://journals.openedition.org/ebisu/5598

DOI : 10.4000/ebisu.5598

ISSN : 2189-1893

Éditeur

Institut français de recherche sur le Japon à la Maison franco-japonaise (UMIFRE 19 MEAE-CNRS)

Édition imprimée

Date de publication : 1 novembre 2021

Pagination : 23-46

ISSN : $1340-3656$

\section{Référence électronique}

Naofumi Nakamura, « Kamaishi face aux crises pluridimensionnelles d'après-guerre. Catastrophes, restructuration industrielle et décroissance démographique », Ebisu [En ligne], 58 | 2021, mis en ligne le 01 novembre 2021, consulté le 14 avril 2022. URL : http://journals.openedition.org/ebisu/5598 ; DOI : https://doi.org/10.4000/ebisu.5598 


\section{Kamaishi face aux crises pluridimensionnelles d'après-guerre.}

Catastrophes, restructuration industrielle et décroissance démographique

NaKamura Naofumi

戦後釜石における危機の多層化: 災害・産業構造転換・人口収縮

中村尚史

Kamaishi in the Face of Post-War Multidimensional Crises.

Disasters, Industrial Reconstruction and Population Decline

NAKAMURA Naofumi

Mots-clés : crise locale, Kamaishi, désastre, restructuration industrielle, décroissance démographique, politique du bricolage

L'auteur : Nakamura Naofumi est professeur à l'Institut des sciences sociales de l'université de Tokyo. Il a obtenu un doctorat en histoire à l'université du Kyūshū en 1997. Ses recherches portent principalement sur l'économie et l'histoire industrielle modernes japonaises.

Résumé : Les communautés locales du Japon moderne font face à des crises pluridimensionnelles : crise chroniques comme la décroissance démographique, progressives comme le déclin des industries de base et soudaines comme les catastrophes naturelles ou les dommages de guerre. C'est cette structure stratifiée qui complique la réponse des régions et la mise en ouvre de mesures appropriées. Cette étude se concentre sur la commune de Kamaishi, dans le département d'Iwate, et vise à examiner l'historique des différentes crises pour mettre en évidence leurs structures à plusieurs niveaux. Nous avons ainsi pu montrer que les habitants sont confrontés, depuis le milieu du $\mathrm{xx}^{\mathrm{e}}$ siècle, à des crises à plusieurs niveaux, auxquelles ils opposent des réponses relevant de l'«ingénierie " et de la politique dite du " bricolage ». 
キーワード

地域の危機、釜石、災害、産業構造転換、 人口減少、ブリコラージュ

著者

中村尚史：東京大学社会科学研究所教授。博 士 (文学) (九州大学、1997 年)。研究分野 は日本経済史・経営史。主な研究テーマは、 近代日本鉄道業の発展に関する研究、地域経 済に関する研究、経営者と労働者のオーラル・ ヒストリー。

要旨

現代日本の地域社会は、人口減少のようにゆ るやかにすすむ慢性的な危機、中核産業の衰
退のような段階的にすすむ危機、自然災害や 戦災のような突発的な危機というように、多 層的な危機に直面している。そして危機の多 層構造が、地域における危機対応を複雑に し、一つの危機への対応がかえつて別の危機 を深刻化させるという事態を招く恐れがある。 本稿では、岩手県釜石市を事例として、地域 における危機の多層構造と、それに対する地 域社会の対応を、歴史的な視点から考えた。 そのことを通して、釜石の人々が 20 世紀半 ば以降、'engineering'的対応と 'bricolage' 的対応をうまく組み合わせつつ、多層的な危 機の構造に立ち向かってきたことが明らかに なった。
Veywords: Local Crisis, Kamaishi Disaster, Industrial Reconstruction, Population Decline, DIY politics.

The Author: Nakamura Naofumi is a professor at the Institute of Social Science at the University of Tokyo. He obtained a $\mathrm{PhD}$ in history from Kyūshū University in 1997. His main area of research is the economic and business history of modern Japan.

Abstract: The local communities of modern Japan are faced with multidimensional crises: chronic crises, such as population decline; gradual crises, like the decline of basic industries; and sudden crises, like natural disasters and war damage. This stratification renders both regional responses and the implementation of measures inappropriate. This research focuses on the city of Kamaishi in Iwate Prefecture, and aims to examine the history of these different crises to foreground their multi-layer structure. In doing so, we have demonstrated how, since the mid-20th century, residents have been confronted with multidimensional crises, and have responded by drawing on "engineering" and "DIY" politics. 


\section{Kamaishi face aux crises pluridimensionnelles d'après-guerre Catastrophes, restructuration industrielle et décroissance démographique}

NAKAmURA Naofumi*

\section{Crise locale pluridimensionnelle}

Cette étude se concentre sur la commune de Kamaishi, dans le département d'Iwate, et vise à mettre en relief, par l'analyse de ses réponses aux crises, les problèmes structurels propres à cette région.

Toute crise locale possède une structure unique qui dépend de divers facteurs : les différences propres aux principaux secteurs d'industrie que sont l'agriculture, la sylviculture et la pêche, ainsi que la production manufacturière; la topographie (montagnes, plaines, littoraux); ou encore la distance aux grandes villes. Les points communs de ces crises locales résident cependant dans leur structure pluridimensionnelle : crises chroniques comme la décroissance démographique, progressives comme le déclin des industries de base et soudaines comme les catastrophes naturelles ou les dommages de

Cet article est la traduction d'une version remaniée, en accord avec l'auteur, de « Sengo Kamaishi ni okeru kiki no tasōka : saigai/sangyō kōzō tenkan/jinkō shūshuku » 戦後 釜石における危機の多層化:災害・産業構造転換・人口収縮，in Genda Yūji 玄田有史 \& Nakamura Naofumi 玄田有史 (dir.), Chiiki no kiki Kamaishi no taiō: tasōka suru kōzō 地 域の危機·釜石の対応: 多層化する構造 (Crise locale et sa réponse à Kamaishi : une structure pluridimensionnelle), Tokyo, Tōkyō daigaku shuppankai 東京大学出版会, 2020.

* Professeur à l'Institut des sciences sociales de l'université de Tokyo. 
guerre. C’est cette structure stratifiée qui complique la réponse des régions aux crises et la mise en œuvre de mesures appropriées.

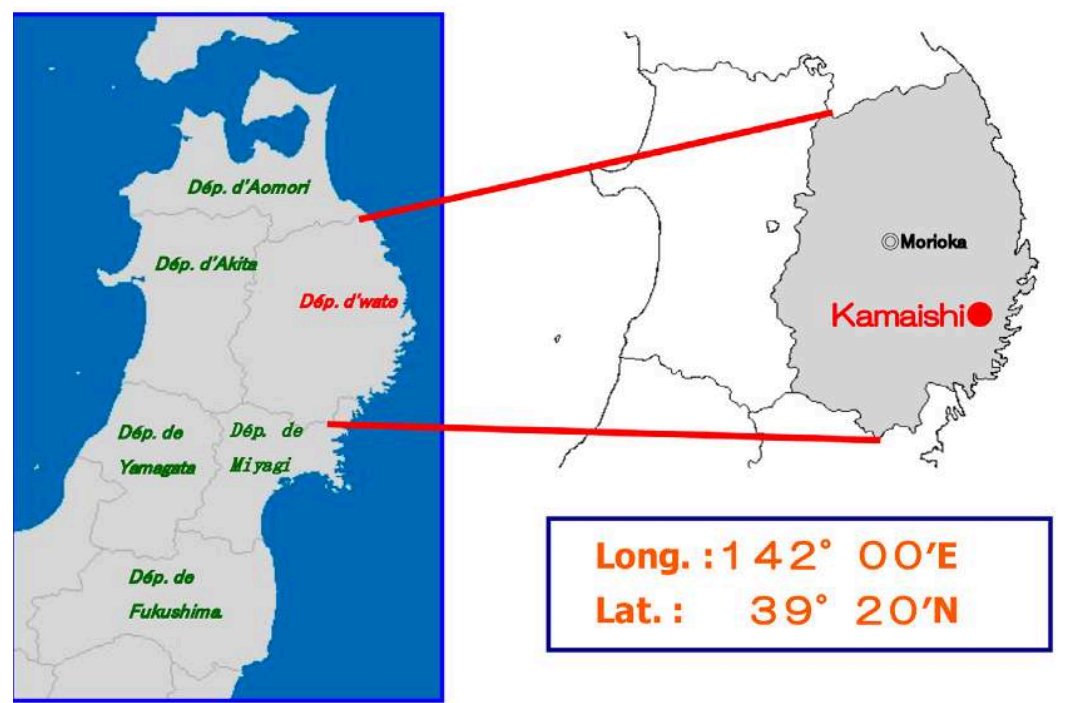

Fig. 01

Situation de la ville de Kamaishi et du département d'Iwate.

La commune de Kamaishi, de tradition industrielle, a autrefois prospéré en tant que siège de l'entreprise Kamashi seitetsusho 釜石製鉄所 (aciérie de Kamaishi) et en raison de la présence de son port de pêche au chalut pélagique. Elle comptait plus de 90000 habitants à son apogée, au début des années 1960. Cependant, en raison du déclin de la sidérurgie et des prises pélagiques, la population a progressivement diminué et Kamaishi ne compte aujourd'hui que 36000 habitants ${ }^{1}$. Après la fermeture du haut fourneau de l'aciérie en 1989, et malgré la stabilisation des industries de base, la décroissance démographique s'est graduellement poursuivie, en particulier dans les années 2000, lors desquelles le taux de croissance démographique a

1. Pour des études antérieures et des enquêtes locales à Kamaishi, voir Nakamura (2009a : 11-14) et Matsuishi (2010). 
augmenté tous les cinq ans (fig. 02). Parallèlement, la part de la population âgée (plus de 65 ans) a dépassé les $30 \%$ tandis que celle des jeunes actifs (de 15 à 29 ans) est tombée à environ $10 \%$, donnant à la ville un air caractéristique de zone dépeuplée. En d'autres termes, si la convergence des secteurs public et privé a réussi à l'emporter sur la crise progressive provoquée par le déclin de l'industrie de base ${ }^{2}$, restait la crise chronique liée à l'exode rural. Puis, en mars 2011, le tsunami provoqué par le grand séisme de la côte Pacifique du Tōhoku a frappé, obligeant la région de Kamaishi à répondre dans les années 2010 à une nouvelle crise, cette fois soudaine.

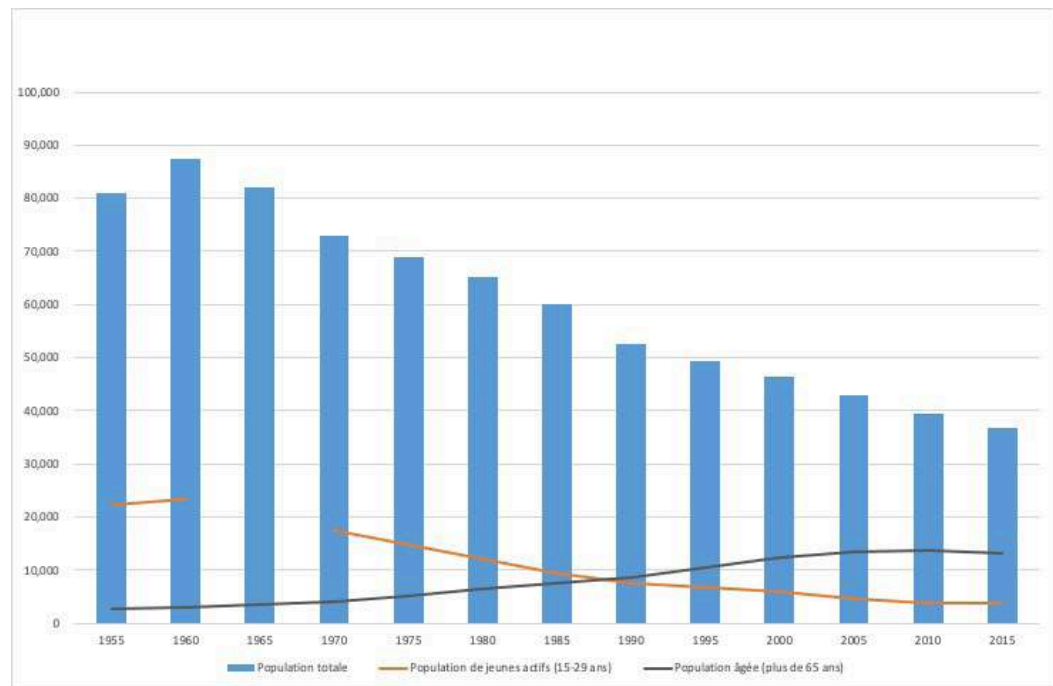

Fig. 02

Évolution de la population à Kamaishi (en nombre de d'habitants).

Sources : Nakamura (2009a : 5); statistiques de la ville de Kamaishi; recensements annuels; Shin Nittetsu gaido 新日鐵ガイド (Guides de l'aciérie Nippon Steel), 2006, 2011 ; rapport annuel 2016 de Shin Nippon Steel; documents de la division de la pêche de la ville de Kamaishi. Remarque : La population a diminué de 60005 personnes entre 1885 et 1990.

2. Une de nos études, fondée sur une enquête sur l'espérance à Kamaishi, a montré qu'une des réponses aux crises chroniques était justement la "renaissance de l'espoir " (Genda \& Nakamura 2009a; Genda \& Nakamura 2009b). 
La ville de Kamaishi représente donc un cas extrême en ce qu'elle combine plusieurs crises : celle, chronique, de l'exode rural, commune à de nombreuses villes de région; celle, progressive, du déclin de plusieurs industries de base; et celle, brusque, du tsunami et de ses dommages. Néanmoins, toutes les régions étant touchées, à des degrés divers, par des crises pluridimensionnelles, le cas de Kamaishi donne un bon exemple de la manière dont les habitants ont fait face, ou ont essayé de faire face à ces crises.

En partant de ce postulat, nous souhaiterions examiner ici l'historique des différentes crises à Kamaishi pour mettre en évidence leur structure pluridimensionnelle.

\section{Une crise soudaine}

«Le tsunami est arrivé, mais [les bombardements navals] pendant la guerre, c'était plus impressionnant encore. "

D’octobre 2011 jusqu'au courant de l'année 2012, nous avons enregistré dans les "Mémoires de l'histoire orale du séisme » les réactions de ceux qui se trouvaient à Kamaishi au moment du tsunami. La phrase ci-dessus est tirée du récit d'une personne ayant fait l'expérience des bombardements navals pendant la seconde guerre mondiale (Tōkyō daigaku shakai kagaku kenkyūjo 2013 : 746). Le paysage de Kamaishi, après le grand séisme de la côte Pacifique du Tōhoku, a en effet été rapproché, non pas des dégâts des tsunamis de 1896 ou de 1933 dans le Sanriku, mais bien de celui façonné par les bombardements de 1945 (fig. 03). En outre, il convient de noter que, derrière les premiers récits, se cachent les sentiments de citoyens se disant qu'ils se sont " remis de ces terribles bombardements navals, donc cette fois-ci aussi, ça ira ». La vue de la ville désertée après les attaques du 14 juillet et du 9 août 1945 rappelle aujourd'hui la rapide reconstruction et la croissance économique qui ont suivi dans la région (Genda \& Nakamura 2020). 


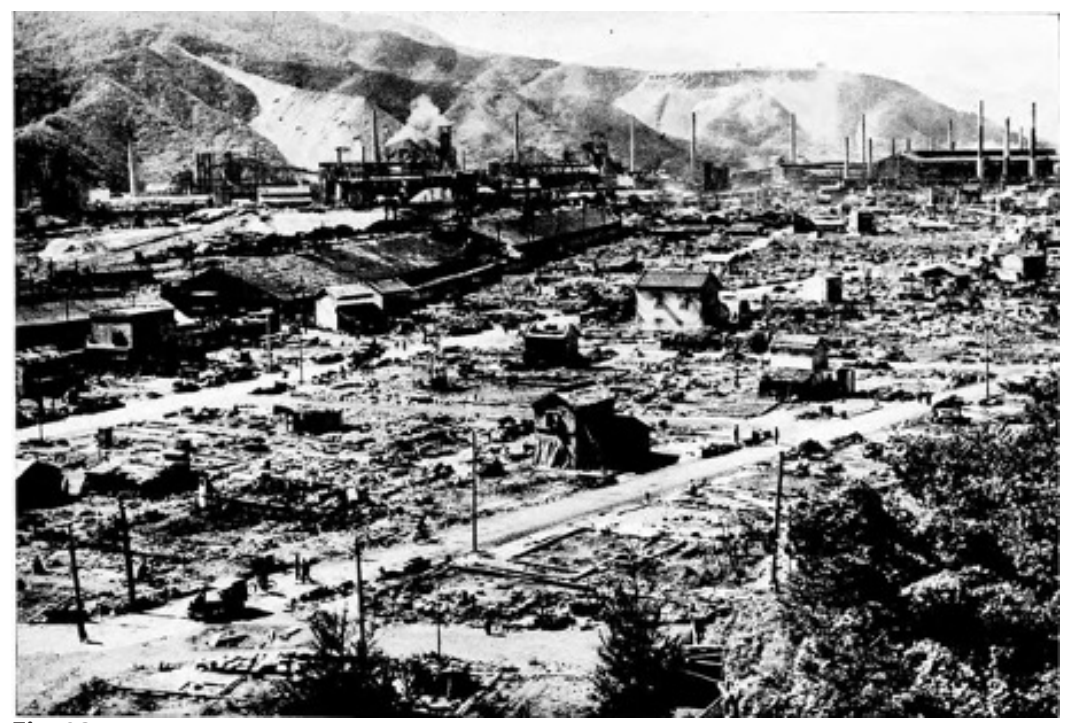

Fig. 03

Centre-ville de Kamaishi après le deuxième bombardement naval, 1945.

Source : «Kamaishi, Naval Bombardments, 14 July and 9 August, Report of Ships' Bombardment Survey Party U.S. Strategic Bombing Survey », p. 172.

Nous avons comparé et examiné l'état des dommages causés par les diverses catastrophes dont la ville de Kamaishi a été soudainement victime (tableau 01). Tout d'abord, nous pouvons noter que les tsunamis d'avantguerre, comme celui de 1896, causaient d'énormes dégâts, avec un taux de mortalité de 53,5\% et un taux de destruction des habitations de 72,5\%. La prise de conscience qui a suivi ce tsunami effroyable de 1896 , qui a partiellement détruit les zones inondées, a incité les habitants à déplacer leurs maisons vers des terrains plus élevés, ce qui a permis de connaitre, dans une certaine mesure, des dégâts moins importants lors du tsunami de 1933. Le taux de mortalité en particulier a considérablement baissé : il était de $1,3 \%$. 


\section{TABLEAU 01}

Tsunamis et bombardements navals dans la région de Kamaishi.

\begin{tabular}{|c|c|c|c|c|c|c|}
\hline Région & & Kamaishi & Hirata & Unosumai & Tōni & Total \\
\hline \multirow{6}{*}{$\begin{array}{c}\text { Tsunami } \\
\text { de Meiji- } \\
\text { Sanriku } \\
\text { de } 1896 \\
\text { (chiffres } \\
\text { du } 15 \text { juin } \\
2014 \text { ) }\end{array}$} & Population d'alors & 5274 & 1255 & 3153 & 2807 & 12489 \\
\hline & Nombre de morts & 3323 & 718 & 1061 & 1585 & 6687 \\
\hline & Taux de mortalité & $63 \%$ & $57,2 \%$ & $33,7 \%$ & $56,5 \%$ & $53,5 \%$ \\
\hline & Nombre de bâtiments & 956 & 149 & 474 & 430 & 2009 \\
\hline & $\begin{array}{c}\text { Nombre de bâtiments } \\
\text { détruits }\end{array}$ & 791 & 107 & 227 & 332 & 1457 \\
\hline & Taux de destruction & $82,7 \%$ & $71,8 \%$ & $47,9 \%$ & $77,2 \%$ & $72,5 \%$ \\
\hline \multirow{7}{*}{$\begin{array}{c}\text { Tsunami } \\
\text { de Shōwa- } \\
\text { Sanriku } \\
\text { de } 1933 \\
\text { (chiffres } \\
\text { du } 15 \text { juin } \\
2014 \text { ) }\end{array}$} & Population d'alors & 24300 & 846 & 2797 & 3694 & 31637 \\
\hline & $\begin{array}{c}\text { Nombre de morts et } \\
\text { disparus }\end{array}$ & 37 & - & 7 & 359 & 403 \\
\hline & Taux de mortalité & $0,2 \%$ & - & $0,3 \%$ & $9,7 \%$ & $1,3 \%$ \\
\hline & Nombre de bâtiments & 4633 & 109 & 402 & 550 & 5694 \\
\hline & $\begin{array}{c}\text { Nombre de bâtiments } \\
\text { détruits }\end{array}$ & 173 & 15 & 141 & 245 & 574 \\
\hline & $\begin{array}{c}\text { Nombre de bâtiments } \\
\text { incendiés }\end{array}$ & 198 & 0 & 0 & 0 & 198 \\
\hline & Taux de destruction & $8 \%$ & $13,8 \%$ & $35,1 \%$ & $44,5 \%$ & $13,6 \%$ \\
\hline \multirow{13}{*}{$\begin{array}{c}\text { Bombar- } \\
\text { dements } \\
\text { navals du } \\
14 \text { juillet } \\
\text { et du } \\
9 \text { août } \\
1945\end{array}$} & $\begin{array}{l}\text { Population d'alors } \\
\text { (juin 1945) }\end{array}$ & & & & & 45815 \\
\hline & $\begin{array}{l}\text { Nombre de morts } \\
\text { (premier bombarde- } \\
\text { ment) }\end{array}$ & & & & & 421 \\
\hline & Dont dans les aciéries & & & & & 106 \\
\hline & $\begin{array}{c}\text { Nombre de morts } \\
\text { (deuxième bombarde- } \\
\text { ment) }\end{array}$ & & & & & 270 \\
\hline & Dont dans les aciéries & & & & & 51 \\
\hline & $\begin{array}{c}\text { Nombre total de } \\
\text { morts }\end{array}$ & & & & & 691 \\
\hline & Taux de mortalité & & & & & $1,5 \%$ \\
\hline & Nombre de bâtiments & & & & & 8330 \\
\hline & $\begin{array}{c}\text { Nombre de bâti- } \\
\text { ments complètement } \\
\text { détruits }\end{array}$ & & & & & 3110 \\
\hline & $\begin{array}{c}\text { Nombre de bâtiments } \\
\text { à moitié détruits }\end{array}$ & & & & & 326 \\
\hline & $\begin{array}{l}\text { Nombre de bâtiments } \\
\text { partiellement détruitss }\end{array}$ & & & & & 685 \\
\hline & $\begin{array}{l}\text { Nombre total de bâti- } \\
\text { ments endommagés }\end{array}$ & & & & & 4121 \\
\hline & Taux de destruction & & & & & $49,5 \%$ \\
\hline
\end{tabular}




\begin{tabular}{|c|c|c|c|c|c|c|}
\hline Région & & Kamaishi & Hirata & Unosumai & Tōni & Total \\
\hline \multirow{5}{*}{$\begin{array}{l}\text { Tsunami } \\
\text { de } 1960 \\
\text { au Chili } \\
\text { (chiffres } \\
\text { du } 24 \text { mai } \\
2014 \text { ) }\end{array}$} & $\begin{array}{c}\text { Nombre de bâtiments } \\
\text { détruits }\end{array}$ & & & & & 11 \\
\hline & \begin{tabular}{|c|} 
Nombre de bâtiments \\
complètement \\
incendiés
\end{tabular} & & & & & 17 \\
\hline & $\begin{array}{c}\text { Nombre de bâtiments } \\
\text { à moitié incendiés }\end{array}$ & & & & & 111 \\
\hline & $\begin{array}{c}\text { Nombre de bâtiments } \\
\text { inondés }\end{array}$ & & & & & 1298 \\
\hline & $\begin{array}{c}\text { Dommage total (en } \\
\text { millions de yens) }\end{array}$ & & & & & 630 \\
\hline \multirow{12}{*}{$\begin{array}{c}\text { Grand } \\
\text { séisme de } \\
\text { la côte Pa- } \\
\text { cifique du } \\
\text { Tōhoku du } \\
11 \text { mars } \\
2011 \\
\text { (chiffres } \\
\text { du } \\
11 \text { mars } \\
2019 \text { ) }\end{array}$} & Population d'alors & 6971 & 6971 & 6630 & 2106 & 39996 \\
\hline & $\begin{array}{c}\text { Nombre de morts et } \\
\text { disparus }\end{array}$ & 229 & 229 & 579 & 21 & 1064 \\
\hline & Taux de mortalité & $3,3 \%$ & $3,3 \%$ & $8,7 \%$ & $1 \%$ & $2,7 \%$ \\
\hline & Nombre de bâtiments & 3291 & 3291 & 2517 & 956 & 16182 \\
\hline & \begin{tabular}{|c|} 
Nombre de bâti- \\
ments complètement \\
détruits
\end{tabular} & & & & & 2957 \\
\hline & \begin{tabular}{|c|} 
Nombre de bâtiments \\
à moitié détruits
\end{tabular} & & & & & 699 \\
\hline & $\begin{array}{l}\text { Nombre de bâtiments } \\
\text { partiellement détruits }\end{array}$ & & & & & 1048 \\
\hline & \begin{tabular}{|c} 
Nombre total de bâti- \\
ments endommagés
\end{tabular} & 1383 & 272 & 1691 & 347 & 4704 \\
\hline & Taux de destruction & $42 \%$ & $21,7 \%$ & $67,2 \%$ & $36,3 \%$ & $29,1 \%$ \\
\hline & $\begin{array}{l}\text { Nombre d'établisse- } \\
\text { ments commerciaux }\end{array}$ & & & & & 2396 \\
\hline & $\begin{array}{l}\text { Nombre d'établisse- } \\
\text { ments commerciaux } \\
\text { détruits }\end{array}$ & & & & & 1382 \\
\hline & $\begin{array}{l}\text { Taux de destruction } \\
\text { des établissements } \\
\text { commerciaux }\end{array}$ & & & & & $57,7 \%$ \\
\hline
\end{tabular}

Sources : Nakamura (2014: 50-53); Kamaishi-shi (2019: 1); Kamaishi-shi-shi hensan iinkai (1977); Kamaishi-shi sensai kiroku hensan iinkai (1976); Bureau des mesures en cas de catastrophe de la ville de Kamaishi « Situation des dommages du grand séisme de la côté Pacifique du Tōhoku de 2011 » (10 avril 2012). Remarques : Les chiffres pour les pertes humaines par région du grand séisme de 2011 datent de la fin de mars 2012, et les chiffres pour les dommages aux bâtiments (habitations unique-

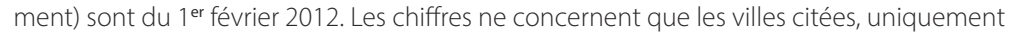
pour les personnes décédées ou disparues dont l'adresse est connue. Les victimes des bombardements navals ne comprennent pas les 32 prisonniers de guerre alliés, les 28 tireurs de canons et les 2 militaires, soit un total de 62 personnes. 
À présent, intéressons-nous aux dommages des bombardements navals. Les pilonnages des forces alliées, du 14 juillet et du 9 août 1945, ont entraîné la mort de 691 personnes, soit un taux de mortalité de 1,5\%, un chiffre donc plus important que le tsunami de 1933 (403 morts), causant également beaucoup de dégâts dans la zone urbaine, avec un taux de destruction de près de $50 \%$. Les Alliés ont bombardé à deux reprises, larguant au total - rien que pour le bombardement effectué par l'armée américaine -3653 obus ( 1605 de 16 pouces et 2048 de 8 pouces) sur la ville de Kamaishi et ses usines ${ }^{3}$. Les obus de gros calibre ont principalement été lancés sur les aciéries, et les autres sur la zone urbaine, les observateurs d'artillerie placés dans les avions s'assurant que les cibles étaient atteintes. Les bombardements du 9 août en particulier se sont poursuivis pendant plus de trois heures, détruisant complètement les principales installations de l'aciérie et incendiant toute la zone urbaine restée intacte après les premiers tirs, y portant ainsi à $71 \%$ le taux de mortalité. Depuis la plate-forme de la gare de Kamaishi à peine endommagée, on pouvait voir, disait-on, la mer de l'autre côté des champs en feu (Nakamura 2009b : 40).

Par ailleurs, le tsunami de 1960 au Chili a démoli pour plus de 600 millions de yens d'habitations, bien qu'aucune perte humaine n'ait été à déplorer à Kamaishi. Cela a permis de lancer la construction de digues dans le cadre de la loi chilienne sur les mesures spéciales de lutte contre les tremblements de terre et les tsunamis (août 1960), encourageant tout au long des années 1960 l'utilisation du béton. Par la suite, est lancée en 1978 la construction du brise-lames le plus profond du monde à l'embouchure de la baie de Kamaishi (63 mètres de profondeur; achevé en 2008), et il semble que les mesures anti-tsunamis aient été renforcées.

Mais le tsunami causé par le grand séisme de la côte Pacifique du Tōhoku du 11 mars 2011 a détruit les digues ainsi que le brise-lames de l'embouchure de la baie et a frappé les zones côtières de la ville. Les pertes humaines s'élèvent à 1064 morts et disparus, soit un taux de mortalité de 2,7\% dépassant de loin les dégâts causés par le tsunami de ShōwaSanriku de 1933 ou les bombardements navals. Le taux de destruction des

3. "Kamaishi, Naval Bombardments, 14 July and 9 August, Report of Ships' Bombardment Survey Party U.S. Strategic Bombing Survey ", RG38 A1 320 (archives nationales des États-Unis). 
établissements commerciaux est également supérieur à celui des bombardements navals (57,7 \%). D'ailleurs, le spectacle des zones inondées, partiellement détruites, en rappelait la tragédie. Mais c'est peut-être justement leur souvenir comme celui de la reconstruction et du développement ultérieurs qui ont été le moteur de l'approche rapide et positive adoptée à l'occasion de la reconstruction à suivre.

\section{Une crise progressive}

"Ville du fer, du poisson et du rugby."

Ce slogan se trouve désormais partout dans la ville. Il est évident que le "fer» désigne ici l'industrie sidérurgique soutenue par les usines de Kamaishi, le "poisson " l'industrie de la pêche pélagique et le "rugby " l'équipe de Shin Nippon seitetsu Kamaishi 新日本製鐵 (Nippon Steel Kamaishi), surnommée "Kita no tetsujin » 北の鉄人 (Hommes de fer du Nord) depuis qu'elle a remporté pendant sept années consécutives le championnat du Japon (entre 1979 et 1985). Ces trois domaines ont en commun d'avoir connu leur apogée dans les années 1970 et 1980, pour ensuite décliner peu à peu et vivoter. De plus, l'expression « ville du fer et du poisson " n'est explicitement utilisée que depuis 2005 environ, soit après que le nombre de salariés de l'aciérie a atteint un minimum de 150 depuis la guerre et après que le volume des transactions du marché aux poissons a chuté au tiers de son niveau le plus élevé ${ }^{4}$ Ainsi, Kamaishi a reconstruit son identité locale en s'appuyant sur la mémoire d'industries autrefois prospères. Mais comment cette ville industrielle a-t-elle fait face à l'affaiblissement progressif de ses industries de base?

4. Une recherche Internet avec les mots «ville du fer et du poisson Kamaishi » a révélé que l'expression est apparue pour la première fois en février 2002 (une seule occurrence) et, depuis 2005, son utilisation est en augmentation principalement par la mairie de Kamaishi. De son côté, l'équipe de rugby de Shin Nippon seitetsu Kamaishi est devenue un club en 2000, le Kamaishi Shīueibusu 釜石シーウェイブス (Kamaishi Seawaves) en 2001, dont le cercle de soutien (de la ville, des entreprises locales et des citoyens) s'est élargi (Miyajima 2009 : 128-139). Là encore, on peut donc dire que le déclin du rugby a participé à la popularité de la " ville du rugby ". 
Tout d'abord, bien que le nombre de salariés de l'aciérie de Kamaishi ait été à son apogée après-guerre, en 1961, soit au cours de la première moitié de la période de la Haute croissance économique (1955-1973) - 13158 personnes employées par l'aciérie principale et les filiales du groupe-, ce nombre a rapidement diminué dès 1964 , avec le transfert à grande échelle vers la nouvelle aciérie de Fuji seitetsu 富士製鐵 située dans la région du Tōkai (Hyaku nen shi hensan iinkai 1986: 239; Nakamura 2009a : 5-6). Cependant, si au début des années 1970, avec les progrès technologiques et la restructuration, la production d'acier brut a continué d'augmenter, malgré une diminution de la main-d'œuvre, dans la seconde moitié des années 1970, lorsque la récession touchant la sidérurgie s'est aggravée, on a assisté à des rationalisations par vagues successives, commencées en 1978, et à la diminution progressive de la production (Hyaku nen shi hensan iinkai 1986 : 266-267). Puis, la principale usine a fermé en 1980 et le haut fourneau en 1989, ce qui mit fin à l'activité de sidérurgie locale, ne laissant subsister qu'une usine de fil machine. En conséquence, le nombre de ses salariés a fortement baissé à partir de 1990, si bien qu'en 2005, il était d'à peine 150 , soit près du centième de son chiffre maximal. Par la suite, ce nombre a cessé de baisser et est remonté à 224 en 2010 (fig. 04). En outre, bien que les installations portuaires et de transport aient été gravement endommagées en mars 2011, les bâtiments des usines ont échappé de justesse à l'impact du tsunami. Par conséquent, le laminage des fils d'acier a repris dès avril et il a été possible d'importer des matières premières et d'expédier des produits grâce aux installations portuaires. La reprise immédiate des affaires a en outre été importante pour maintenir les commandes et rendre attractif le maintien de l'aciérie de Kamaishi dans le département, mais aussi à l'échelle nationale (Shin Nippon seitetsu Kamaishi seitetsujo 2014 : 382-389). Depuis, le nombre de salariés a progressivement augmenté pour atteindre 237 en 2015.

Voyons ce qu'il en est du volume des transactions sur le marché aux poissons. La ville, devenue un centre de la pêche au thon dans les mers de Béring et d'Okhotsk après-guerre, se vantait d'un volume d'affaires de 10 à 11 milliards de yens au début des années 1980, période pendant laquelle elle profitait du prix élevé du poisson, de l'augmentation des stocks de sardines, mais également de l'essor de la pêche pélagique au calmar. Cependant, la mise en place du système des 200 milles marins (zone économique exclusive) en 1977 a entraîné la réduction de zones de pêche, puis le démantèlement 
de la pêche pélagique et, dans la seconde moitié des années 1980, c'est au tour de la pêche hauturière de décliner, car elle est devenue non rentable en raison de la baisse du prix du poisson et de la hausse du prix du fioul, toutes deux liées à l'augmentation rapide des importations de produits de la mer. En conséquence, le rendement des transactions du marché aux poissons de Kamaishi tombe de 7,9 milliards de yens en 1990 à 4,2 milliards de yens en 1995 (Kase 2008 : 89, 2009 : 204-205). Dans les années 2000, il plafonne à 3 milliards de yens à peine, mais le tsunami causé par le grand séisme de la côte Pacifique ayant gravement endommagé la pêche côtière, il chute à 1,77 milliard de yens en 2015 (fig. 04).

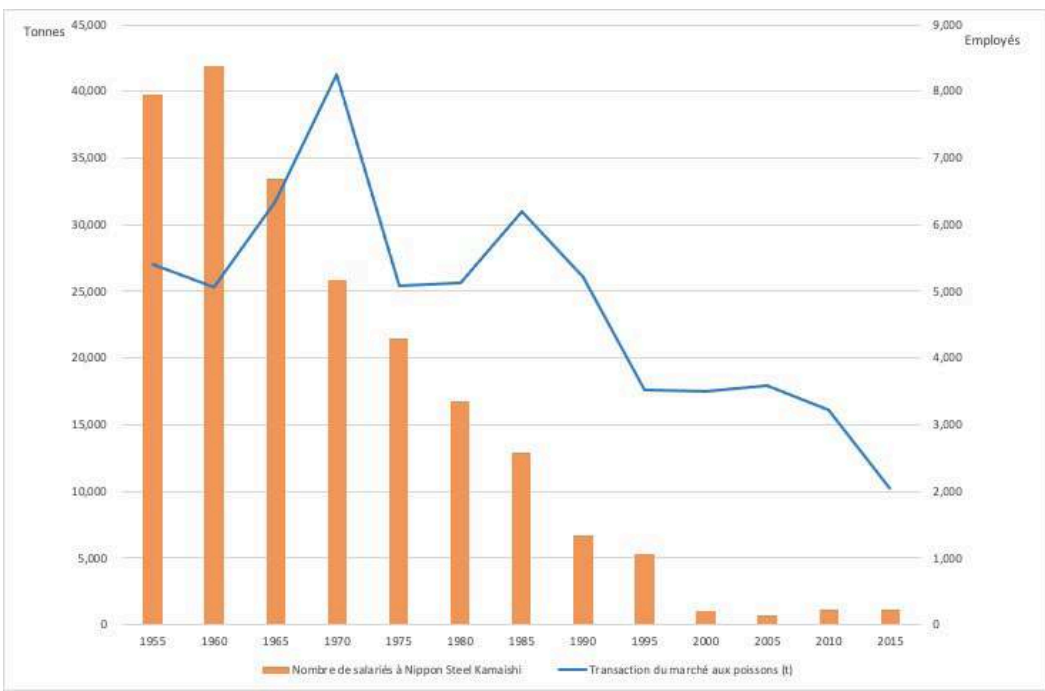

Fig. 04

Nombre de salariés à Nippon Steel Kamaishi et volume de transactions du marché aux poissons de Kamaishi.

Sources: Nakamura (2009a : 5); statistiques de la ville de Kamaishi; recensements annuels; guides de l'aciérie Nippon Steel, 2006, 2011; rapport annuel 2016 de Shin Nippon Steel; documents de la division de la pêche de la ville de Kamaishi. Remarque : La population a diminué de 60005 personnes entre 1885 et 1990.

Comme décrit ci-dessus, l'industrie sidérurgique de Kamaishi et la pêche ont respectivement atteint leur apogée au début des années 1970 et au début des années 1980, pour décliner depuis lors. Cependant, il convient de noter que les habitants ne sont pas restés les bras croisés face au déclin 
des grandes industries : ils ont activement pris des mesures pour répondre à cette crise progressive et pour maintenir la communauté locale. En freinant les investissements excessifs dans les secteurs de la pêche et des produits manufacturés pendant la croissance du début des années 1980, il a été possible, après les années 1990, de répondre à la dégradation rapide de l'environnement, et de suivre un processus relativement graduel de réduction de la pêche (Kase $2008: 89)^{5}$. Depuis la seconde moitié des années 1970, les aciéries se sont quant à elles appliquées à favoriser le développement technologique et à renforcer les usines de fil machine, afin de préserver la survie du bureau de Kamaishi (Aoki, Nitta \& Umezaki 2009 : 79-85). En outre, la ville a fourni un effort tout particulier - notamment à travers la création ou l'invitation d'entreprises nouvelles, en coopération avec les aciéries locales - pour préserver les emplois dans la région (Nakamura 2009 : 152165). Ces efforts constants avaient pour but de freiner le rapide déclin de l'économie locale dû à la chute des aciéries.

En conséquence, les produits manufacturés de Kamaishi destinés à l'exportation ont connu une certaine revalorisation après un creux en 1990, à la suite de l'arrêt du haut fourneau, et ont fortement augmenté de 2005 à 2008 (fig. 05). Cela reflète une bonne performance de la production de fil d'acier et l'attractivité des entreprises, grâce aux efforts de ceux qui ont affronté cette " crise progressive ». La valeur des produits manufacturés, en cours de reprise, a chuté une fois en 2009 en raison de la crise financière de 2008, avant d'être de nouveau touchée par le grand séisme de mars 2011. Cependant, elle est en hausse constante depuis 2012, et est restée stable de 2013 à 2016 malgré la stagnation de l'économie japonaise. Si l'on considère la répartition par branche d'activité, l'acier, qui représentait 70,9\% du total en 1981, a fortement baissé depuis 1990 en raison du déclin des aciéries, atteignant $26 \%$ en 2000 . Au contraire, le nombre de machines chargées d'attirer les entreprises a augmenté, atteignant 36,1 \% en 2000 (Nakamura 2009; fig. 06). Mais depuis 2005, la part de l'acier a augmenté, pour revenir à 53,3 \% en 2008. De plus, de 2011 à 2012, la valeur totale des exportations de produits manufacturés a chuté après la triple catastrophe et a été

5. Ici, Kamaishi est comparé à Hachinohe et à sa croissance rapide, suivie d'un déclin dû à une forte baisse du prix des débarquements, passant de 90 milliards de yens à 10 milliards de yens au cours de la période. 
soutenue par l'acier, dont le niveau se maintient à environ $55 \%$. Toutefois, depuis lors et jusqu'à aujourd'hui, cette part a légèrement diminué en raison de la faiblesse des livraisons et de la progression des machines et des produits alimentaires ${ }^{6}$.

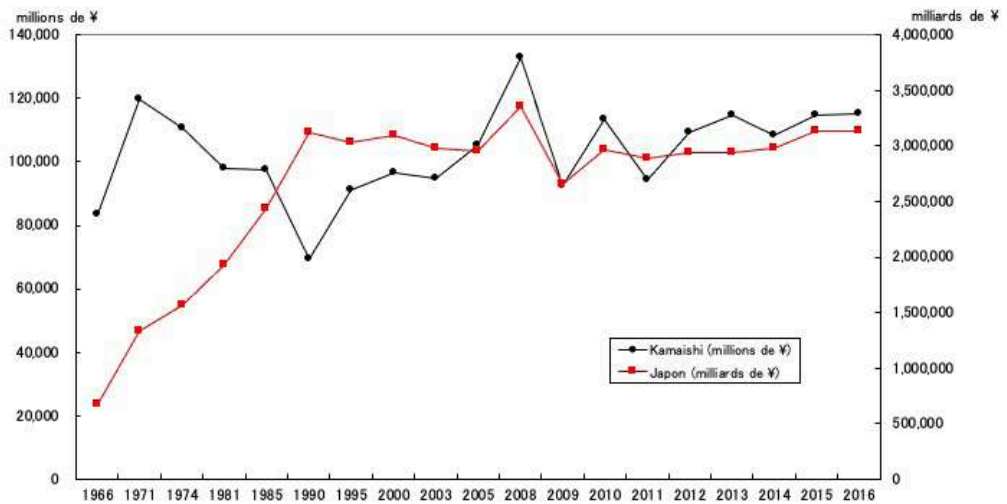

\section{Fig. 05}

Évolution de la valeur des produits manufacturés (en termes réels rapportés à l'indice des prix des biens, base 100 en 2015).

Sources : statistiques de la ville de Kamaishi; études sur les produits.

6. À partir de 2016, la part du prix des exportations de produits manufacturés se répartit comme suit : acier à 39,3\%, machines à 32,8\% et produits alimentaires à $15 \%$. Ces chiffres viennent de l'enquête sur les statistiques de la ville de Kamaishi et des statistiques industrielles publiées chaque année. 


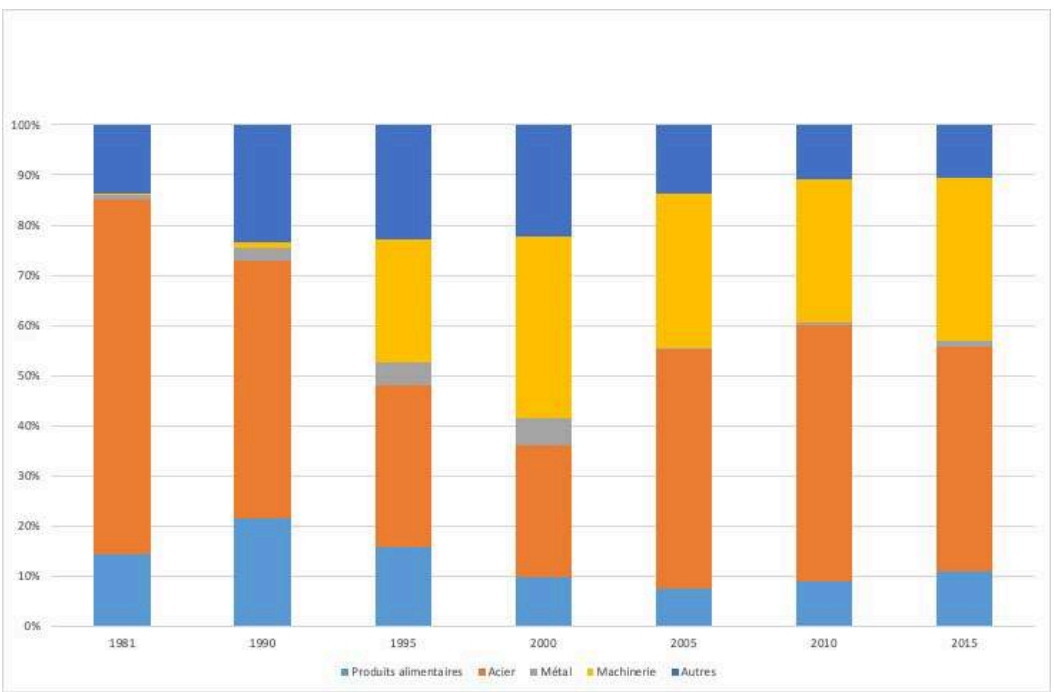

Fig. 06

Répartition des productions des industries manufacturières de Kamaishi.

Sources : Nakamura 2009a : 6; statistiques de la ville de Kamaishi, 2010-2017; enquêtes sur les statistiques industrielles, 2011-2012.

Remarque : Seuls les établissements commerciaux de 4 personnes ou plus sont pris en compte. Les blancs représentent les établissements commerciaux qui gardent leurs chiffres secrets.

\section{Une crise chronique}

L'exode rural dû à la décroissance démographique est considéré comme représentatif des crises locales. La loi sur les mesures spéciales pour la promotion de l'indépendance dans les zones dépeuplées (kaso chiiki jiritsu sokushin tokubetsu sochi hō 過疎地域自立促進特別措置法, promulguée en mars 2000, amendement définitif en mars 2017) détaille dans son article 2, paragraphe 1 , les critères des zones dépeuplées ainsi qu'une liste des municipalités y répondant au paragraphe 2 . Selon ces critères, le ministère des Affaires intérieures et des Communications (Sōmushō 総務省, MIC) a défini (en avril 2017) 647 municipalités comme zones dépeuplées, dont 
Kamaishi, sur les 1718 que compte le pays ${ }^{7}$. En d'autres termes, on peut dire que Kamaishi partage cette crise avec 646 autres municipalités considérées comme dépeuplées, dans tout le pays. Voyons à présent en détail la progression du dépeuplement dans la ville de Kamaishi (fig. 07).

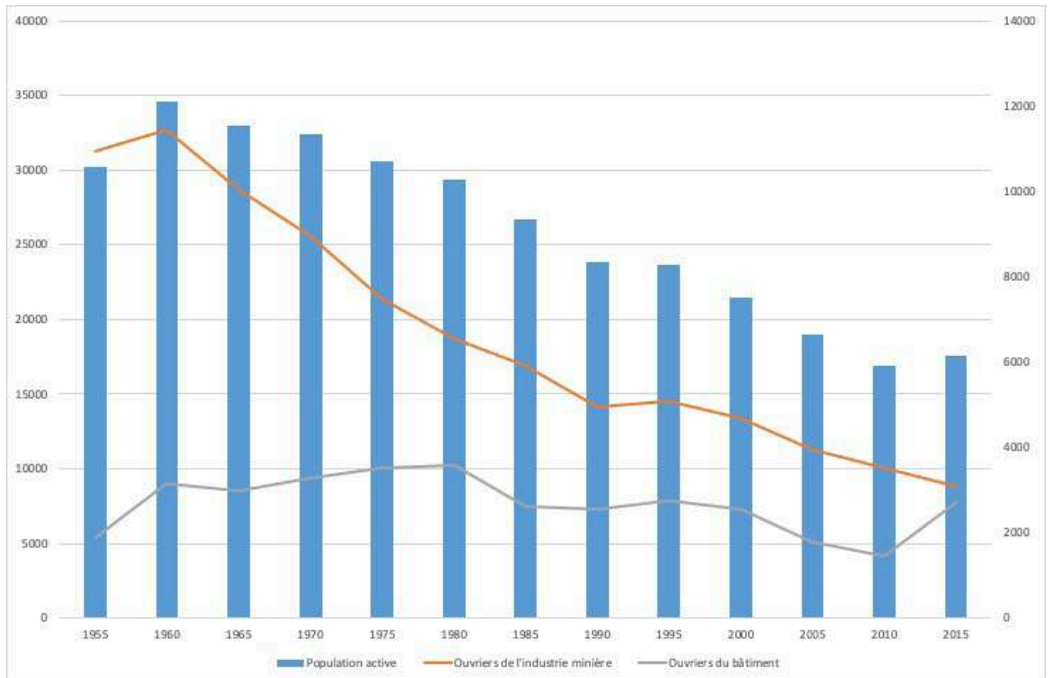

Fig. 07

Évolution de la répartition de la population active.

Source : recensement du Japon; statistiques de la ville de Kamaishi.

Le recensement national indique que la population atteint un pic de 87511 habitants en 1960, mais, selon la revue éditée par la municipalité, la population aurait atteint 91664 habitants en 1961 (Kamaishi-shi-shi hensan iinkai 1977). Cependant, en raison de la migration progressive des salariés et de leurs familles de l'aciérie de Kamaishi à celle de Tōkai, la population a chuté à 72923 en 1970, et diminue depuis continuellement à cause de la restructuration de l'aciérie (Nakamura 2009a : 5-6). Depuis la fermeture du haut fourneau en 1989, la tendance s'est soudainement accélérée,

7. Voir les mesures contre le dépeuplement administratif et financier local sur la page d'accueil du ministère des Affaires intérieures et des Communications: http://www. soumu.go.jp/main_content/000476763.pdf. 
et la population est tombée en dessous des 50000 habitants en 1995, puis en dessous de 40000 en octobre 2010, soit juste avant le grand séisme de 2011. La ville avait alors un pourcentage de jeunes actifs de 9,8\% (le taux minimum indiqué par la loi sur les zones dépeuplées est de $12 \%$ ); celui des personnes âgées était de 34,8\% (32\% ou plus selon la loi) et la décroissance démographique de $34 \%$ pour la période 1985-2010 (19\% ou plus selon la loi) : elle avait ainsi tous les critères d'une zone dépeuplée. La fermeture du haut fourneau en 1989 a clos la période de déclin démographique et social marqué par la contraction du noyau industriel local, et par la suite, la décroissance démographique est due essentiellement au solde naturel. Pendant cette période en particulier, le taux de la population jeune est tombé en dessous de $20 \%$ en raison du manque d'emploi, et la population a continué de vieillir.

Après le grand séisme de la côte Pacifique du Tōhoku, la population a encore diminué, mais la part active, en baisse constante jusqu'alors, a cessé de diminuer. Le nombre d'ouvriers du bâtiment et les flux migratoires venant d'autres villes et villages du département, ainsi que de tout le pays, ont rapidement augmenté. On peut penser qu'il s'agit ici d'une augmentation temporaire due aux projets de reconstruction. Par conséquent, une fois ceux-ci achevés, la population risque de décliner à nouveau.

\section{Des réponses aux crises locales sont-elles possibles?}

Dans le présent article, nous avons analysé selon trois angles - crises soudaine, progressive et chronique - la structure pluridimensionnelle des crises dans la région de Kamaishi. Toutefois, comment y ont réagi les habitants? Pour répondre à cette question, nous aimerions examiner l'histoire et l'état actuel de la réponse à ces crises, ainsi que les problèmes à venir.

Tout d'abord, examinons les caractéristiques des réponses données par la ville à une crise soudaine, en comparant l'historique des reconstructions après la guerre et après le grand séisme de la côte Pacifique du Tōhoku. En septembre 1945, juste après la guerre, le conseil municipal de la ville, complètement détruite par deux bombardements navals, décide de construire 1000 maisons préfabriquées dans le cadre du programme de reconstruction d'après-guerre (sensai fukkō kan.i jūtaku 戦災復興簡易住宅, pour un montant de 660000 yens de l'époque), et il lance dans un premier temps 
le travail de déblayage des gravats (36542 yens ont été apportés par l'État pour un coût total de 54785 yens). Par ailleurs, il approuve en octobre de l'année suivante un budget spécial pour la construction de logements à la suite des dommages de guerre (pour un total de 2,6 millions de yens). Ainsi, la ville de Kamaishi commence à reconstruire, à un rythme soutenu, des habitats immédiatement après la fin de la guerre. Elle poursuit alors ses activités en collectant les fonds nécessaires, grâce par exemple à des emprunts auprès des banques, sans attendre de subvention de la part du gouvernement central (Kamaishi-shi sensai kiroku hensan iinkai 1976 : 115-120). Une telle reconstruction fondée sur des ressources financières indépendantes a certes permis une reprise rapide, mais elle a augmenté le fardeau financier de la ville et aurait pu conduire, à long terme, à une crise financière. Selon une étude d'Uno Shigeki 宇野重規, l'une des causes lointaines de la crise financière qui a éclaté vers 1955 était justement l'énorme charge des coûts de reconstruction (Uno 2009 : 122) ${ }^{8}$.

De même, après le grand séisme de la côte Pacifique du Tōhoku, la ville de Kamaishi s'est de nouveau orientée vers une reconstruction rapide. Selon Araki Kazuo 荒木一男, elle a conçu un grand projet de reconstruction immédiatement après le séisme et a activement collecté dans ce cadre des subventions auprès du gouvernement central. Cela a eu pour conséquence la réduction des dépenses ordinaires et l'amélioration de sa situation financière ${ }^{9}$. Contrairement à l'après-guerre, où Kamaishi ne pouvait pas compter sur les subventions de l'État à cause de l'effondrement des finances nationales dû à la défaite, la ville a pu bénéficier après le grand séisme de 2011 d'un grand soutien national. On peut dire que ces deux projets de reconstruction ont en commun des mesures prises immédiatement après les catastrophes. Araki explique cette rapide réaction comme résultant de la capacité à répondre aux crises financières répétées auxquelles la ville de Kamaishi a dû faire face ${ }^{10}$. Les différents contextes de ces crises financières - soudain avec les bombardements navals, progressif avec le déclin de

8. Discours de M. Mori Shinichirō 森慎一郎, devenu directeur financier de la ville de Kamaishi après la guerre (Genda 2009 : 79-80). À partir de 1955, pour une échelle financière de 437 millions de yens, il semblerait qu'il y eût un déficit de 164 millions de yens (Kamaishi-shi-shi hensan iinkai 1977 : 634-635).

9. Voir le chapitre 3 du livre d'où est tirée cette traduction.

10. Idem. 
l'aciérie, chronique avec la décroissance démographique -, ont permis à la ville de développer sa capacité de réponse aux crises pluridimensionnelles.

Concentrons-nous à présent sur la crise progressive. Les réponses discontinues sur une longue période à la crise progressive liée au déclin des industries de base à Kamaishi ont permis de maintenir la présence du « fer et du poisson ». En outre, la valeur des produits manufacturés en acier exportés a recommencé à augmenter à l'époque du grand séisme de la côte Pacifique du Tōhoku, et celle des produits alimentaires, notamment halieutiques, s'est rétablie depuis $2013^{11}$. De plus, des transformateurs secondaires de produits halieutiques à haute valeur ajoutée, tels que Ono shokuhin 小野食品, ont vu le jour, et l'industrie des produits maritimes transformés se développe également de façon régulière (Nakamura 2019). C'est dans ce contexte qu'est apparue l'expression «ville du fer et du poisson », et, grâce à l'enregistrement en 2015 du haut fourneau au patrimoine mondial, qu'elle est devenue l'identité locale dominante. Il reste cependant quelques problèmes dans l'aménagement urbain autour du patrimoine passé de cette " ville du fer et du poisson ".

Tout d'abord, la culture du travail selon laquelle d' « excellents travailleurs sont prêts à travailler jour et nuit en trois équipes ", " patrimoine " de la sidérurgie et expression-clé pour attirer les entreprises à Kamaishi, est aujourd'hui au bord de l'effondrement (Nakamura 2009 : 163-164). Kamaishi a désespérément absorbé dans l'économie locale l'excédent de personnel généré par la suppression du haut fourneau. De plus, le recrutement extrêmement restreint des années 1990 au début des années 2000 dans l'aciérie alors restructurée, particulièrement faible de 1995 à 2003, a faussé la structure par tranches d'âge ${ }^{12}$. À cela s'ajoutent les départs en retraite à partir de 2015 (à 65 ans) de ceux qui avaient environ 40 ans vers 1990 et représentaient la force de travail. Enfin, la pénurie de maind'œuvre après le grand séisme de la côte Pacifique du Tōhoku a aggravé une situation où la formation de travailleurs qualifiés a rendu difficile la transmission des compétences et de la culture du travail, et la part de travailleurs

11. La part des produis alimentaires, de $6,3 \%$ en 2008, est passée à 12,2 \% en 2013 et à $15 \%$ en 2016 (selon les statistiques de la ville de Kamaishi).

12. Voir le chapitre 6 du livre d'où est tirée cette traduction. 
qualifiés (qui faisaient la force de Kamaishi) a diminué. Cela peut, à terme, conduire directement à diminuer l'attractivité de la région.

En revanche, du fait de la reconnaissance renouvelée de l'identité locale de Kamaishi en tant que "ville du poisson ${ }^{13}$ ", la reconstruction du marché aux poissons s'est imposée après le séisme comme un symbole indispensable. Les capitaux investis en prévision de retombées économiques externes - comme le tourisme - et les projets de développement futur dépassaient alors la demande réelle, de sorte que pour le moment, le montant des transactions n'est pas suffisant. Cela risquerait d'entraîner une perte du " patrimoine " associé à la pêche traditionnelle de Kamaishi, qui avait jusqu'alors évité un net déclin en freinant ces investissements excessifs.

Enfin, le dernier point à discuter est la réponse donnée à la crise chronique de la décroissance démographique. Il semble indispensable, en termes de sécurisation de l'emploi, de poursuivre les efforts de création d'emplois en misant sur l'attractivité des autres entreprises toujours actives malgré le déclin de l'aciérie de Kamaishi. Avant le séisme, la ville en comptait douze, mais six ont été endommagées par le tsunami (Kamaishi-shi 2019: 15). Parmi celles-ci, deux ont fermé, mais quatre ont reconstruit leurs bureaux et sont restées à Kamaishi. Sept nouvelles entreprises ont été accueillies et six autres ont pénétré le marché de manière indépendante, portant le total à vingt-trois entreprises. Parmi ces treize nouvelles entreprises, cinq sont issues de l'industrie des produits maritimes transformés - la reconstruction des entreprises détruites et la revitalisation de la chaîne d'approvisionnement des produits de la mer - et quatre de l'industrie de la logistique et du transport - représentative de la commodité du transport à Kamaishi. Comme le soutient Kikkawa Takeo 橘川武郎, le développement du port et l'ouverture de l'autoroute nationale après le séisme ont fait de Kamaishi un centre logistique avantageux de la côte du Sanriku ${ }^{14}$. On peut dire qu'une utilisation efficace des infrastructures pourrait être la clé du développement économique futur de Kamaishi.

Par ailleurs, si la ville souhaite reconstruire son identité locale sous le slogan "ville du fer, du poisson et du rugby", il est alors légitime de se demander quelle forme elle compte donner au "fer ", au "poisson » et

13. Voir le chapitre 13 du livre d'où est tirée cette traduction.

14. Voir le chapitre 7 du livre d'où est tirée cette traduction. 
au « rugby ». Comme mentionné ci-dessus, le «fer» et le "poisson » parviennent à peine à garder leur rôle d'industries de base; leur avenir est incertain et les problèmes nombreux. Par ailleurs, le "rugby " dépendant trop de la Coupe du monde, il est difficile, pour le moment, de le considérer comme une industrie de base. La tendance à l'augmentation des flux de personnes autour de ces trois jalons montre qu'il est possible de les utiliser comme ressources touristiques. Cependant, alors que le nombre de touristes dans la région avant le séisme était d'environ 800000 (années 200910), il a baissé de 2011 à 2016 pour stagner entre 200000 et 300 000, sans augmenter depuis (Kamaishi-shi 2018 : 10). En outre, bien que l'industrie du tourisme soutienne une plus grande circulation des populations, avec des bénéfices importants sur l'économie, elle manque de stabilité pour devenir une industrie de base.

En revanche, si la lente décroissance démographique est inévitable, il est nécessaire d'envisager la reconstruction des espaces urbains en villes compactes. L'incapacité des finances des collectivités locales à supporter les coûts d'entretien des infrastructures sociales et des services administratifs et médicaux, en raison du vieillissement de la population, est un problème courant dans les municipalités dépeuplées. Face à cela, la ville de Kamaishi a regroupé dans le centre-ville endommagé 430 des 1316 logements sociaux après la catastrophe ${ }^{15}$ et, en densifiant les fonctions urbaines, a réussi à créer un espace urbain étonnamment compact ${ }^{16}$. Ce sera donc un facteur important dans l'étude future de la réponse à la crise chronique à venir.

La ville de Kamaishi fait face à des crises pluridimensionnelles depuis plus de 100 ans. Elle a de nouveau été gravement endommagée par le typhon $n^{\circ} 19$ qui a frappé l'Archipel les 12 et 13 octobre 2019, détruisant 260 maisons, coupant plusieurs routes et détériorant des établissements de transport et de commerce comme le chemin de fer du Sanriku ${ }^{17}$. Cela

15. "État de l'entretien des logements publics de reconstruction de la ville de Kamaishi ", ville de Kamaishi, 21 décembre 2018. http://www.city.kamaishi.iwate.jp/fukko_joho/ fukko_juutaku/ayumi/_icsFiles/afieldfile/2019/01/30/shinchokujyoukyou20181221.pdf.

16. La ville de Kamaishi est à l'origine de "Front Projects 1-3 ", de grands projets pour la revitalisation du quartier est et du centre-ville, et le soutien du gouvernement a permis sa réalisation immédiatement après le séisme (Kamaishi-shi 2019 : 17-19).

17. «Reiwa gan.nen taifū 19gō ni tomonau saigai ni kakaru higai jōkyō nado ni tsuite» 令和元年台風19号に伴う災害に係る被害状況等について (État des dégâts liés au 
a eu pour conséquence l'annulation du match de la Coupe du monde de rugby entre le Canada et la Namibie, prévu le 13 octobre de la même année, mettant aussi la ville face à une crise liée aux événements sportifs. En revanche, la profonde mobilisation face aux pertes humaines liées aux catastrophes a convaincu les athlètes et les spectateurs de s'engager dans du bénévolat. Les images des joueurs canadiens se consacrant au nettoyage de la boue ont été diffusées dans le monde entier, portant à la connaissance de tous "Kamaishi ", la ville qui a survécu à des crises répétées.

Quelle que soit la crise, Kamaishi se relèvera sans peur, prête à prendre une voie nouvelle pour son avenir. Sa longue histoire et son expérience des crises en font un modèle à prendre en compte lorsqu'il s'agit de répondre aux crises locales.

Traduit du japonais par Amira ZEGROUR.

typhon $n^{\circ} 19$ de 2019), ville de Kamaishi, 30 octobre 2020, 17 h. http://www.city. kamaishi.iwate.jp/kurasu/bosai_saigai/saigai_joho/detail/_icsFiles/afieldfile/2019/10/31/ R1T19_201910301700HP.pdf. 


\section{Bibliographie}

AoKı Hiroyuki 青木宏之, NITTA Michio 仁田道夫 \& UMEZAKI Osamu 梅崎修 2009 "Soshiki no kibō Kamaishi seitetsusho no kako to genzai » 組織の希望 釜 石製鉄所の過去と現在 (Espoir d'une entreprise. Passé et présent de l'aciérie de Kamaishi), in Genda Yūji 玄田有史 \& Nakamura Naofumi 中村尚史 (dir.), Kibōgaku 2. Kibō no saisei 希望学2 希望の 再生 (Études sur l'espoir 2. Renaissance de l'espoir), Tōdai shaken 東大社研, Tokyo daigaku shuppankai 東京大学 出版会: 62-103.

\section{Genda Yūji (dir.) 2009}

Kibō o meguru taiwa 希望をめぐる対話 (Dialogue sur l'espoir), Tōkyō daigaku shakai kagaku kenkyūjo 東京大学社会科 学研究所 (Institut des sciences sociales de l'université de Tokyo), 34.

\section{Genda Yūji \& Nakamura Naofumi 2009a} Kibōgaku 2. Kibō no saisei, Tōdai shaken, Tokyo daigaku shuppankai.

\section{Genda Yūji \& NaKamura Naofumi 2009b}

Kibōgaku 3. Kibō o tsunagu 希望学3 希望 をつなぐ (Études sur l'espoir 3. Connecter l'espoir), Tōdai shaken, Tokyo daigaku shuppankai.

\section{Genda Yūji \& NaKamura Naofumi 2014} "Mochiba » no kibōgaku. Kamaishi to shinsai, mōhitotsu no kioku <持ち場>の希 望学 釜石と震災、もう一つの記憶 (Études sur les aspirations professionnelles. Kamaishi et le séisme, un autre souvenir), Tōdai shaken, Tokyo daigaku shuppankai.

Hyaku nen shi hensan iinkai 百年史編纂 委員会 (Comité des 100 ans d'histoire) (dir.) 1986

Tetsu to tomo ni hyaku nen shashin shiryō 鐵と共に百年 写真·資料 (Cent ans de photographies de fer), Shin Nippon seitetsu kabushiki gaisha Kamaishi seitetsujo 新日本製鐵株式会社釜石製鐵所 (aciérie Nippon Steel Kamaishi).

Kamaishi-shi 釜石市

(Ville de Kamaishi) 2018

«Kamaishi-shi suisan shinkō bijon » 釜石市水産振興ビジョン (Vision pour la promotion de pêche de la ville de Kamaishi).

https://www.city.kamaishi.iwate.jp/ docs/2018122000035/

\section{Kamaishi-shi 2019}

«Tawamazu kussezu fukkyū/fukkō no ayumi. Iwate-ken Kamaishi-shi » 撓まず 屈せず:復旧·復興の歩み 岩手県釜石市 (Étapes pour un rétablissement/une reconstruction sans plier de la ville de Kamaishi).

https://www.city.kamaishi.iwate.jp/ docs/2019060400109/file_contents/ ayumi202103.pdf

Kamaishi-shi sensai kiroku hensan iinkai 釜石市戦災記録編纂委員会 (Comité d'archivage des registres des dégâts de guerre de la ville de Kamaishi) (dir.) 1976

Kamaishi kanpō sensai-shi 釜石艦砲 戦災誌 (Revue des bombardements navals de Kamaishi). 
Kamaishi-shi-shi hensan iinkai 釜石市誌 編纂委員会 (Comité de compilation des revues de Kamaishi) (dir.) 1977 Kamaishi-shi-shi tsūshi 釜石市誌 通史 (Histoire des revues de la ville de Kamaishi).

\section{KASE Kazutoshi 加瀬和俊 2008}

« Kamaishi-shi ni okeru gyogyō » 釜石 市における漁業 (Pêcherie dans la ville de Kamaishi), Shakai kagaku kenkyū 社会科 学研究 (Recherche en sciences sociales), 59 (2) : 85-104.

\section{KASE Kazutoshi 2009}

« Kazoku jiei gyogyō ni okeru kibō to jisei » 家族自営漁業における希望と自制 (Espoir et maîtrise de soi dans la pêche familiale indépendante), in GendA Yūji \& Nakamura Naofumi (dir.), Kibōgaku 2. Kibō no saisei, Tōdai shaken, Tokyo daigaku shuppankai : 201-228.

\section{MatsuISH Yasuhiko 松石泰彦 2010}

Kigyō jōkamachi no keisei to Nihonteki keiei 企業城下町の形成と日本的経営 (Création d'une ville de tradition industrielle et gestion à la japonaise), Tokyo, Dōseisha 同成社.

\footnotetext{
MiYaJima Yoshiaki 宮島良明 2009

«Supōtsu ni yoru chiiki saisei no kanōsei. Kamaishi ni okeru ragubī e no kitai to genjitsu » スポーツによる地域再 生の可能性 釜石におけるラグビーへの期 待と現実 (Possibilité de revitalisation locale par le sport. Attentes et réalité du rugby à Kamaishi), in Genda Yūji \& NaKamura Naofumi (dir.), Kibōgaku 3 kibō o tsunagu, Tōdai shaken, Tokyo daigaku shuppankai : 119-143.
}

\section{NaKamura Keisuke 中村圭介 2009} « Kigyō yūchi to jiba kigyō no jiritsu » 企業誘致と地場企業の自立 (Attirer les entreprises et indépendance des entreprises locales), in GendA Yūji \& Nakamura Naofumi (dir.), Kibōgaku 2. Kibō no saisei, Tōdai shaken, Tokyo daigaku shuppankai : 145-196.

\section{NaKamura Naofumi 2009a}

«Kamaishi de kibō o kangaeru kibōgaku Kamaishi chōsa no gaiyō » 釜石で希 望を考える 希望学·釜石調査の概要 (Penser l'espoir à Kamaishi. Études sur l'espoir, compte rendu d'enquête à Kamaishi), in Genda Yūji \& NaKamURA Naofumi (dir.), Kibōgaku 2. Kibō no saisei, Tōdai shaken, Tokyo daigaku shuppankai : 2-23.

\section{Nakamura Naofumi 2009b}

« Kioku no genryū. Kamaishi chiiki no kindai-shi » 記憶の源流 釜石地域の近代史 (Les origines de la mémoire. Histoire moderne de la région de Kamaishi), in Genda Yūji \& Nakamura Naofumi (dir.), Kibōgaku 2. Kibō no saisei, Tōdai shaken, Tokyo daigaku shuppankai : 27-57.

\section{Nakamura Naofumi 2014}

« Kamaishi ni okeru shinsai no kioku » 釜石における震災の記憶 (Souvenirs du tremblement de terre à Kamaishi), in Genda Yūji \& Nakamura Naofumi (dir.), "Mochiba » no kibōgaku, Tōdai shaken, Tokyo daigaku shuppankai : 45-111.

\section{NaKamura Naofumi 2019}

« Kiki o tenki ni kaeru Kamaishi ni okeru hisai kigyō no kiki taiō» 危機を転機に 変える 釜石における被災企業の危機対応 
(Faire de la crise un tournant. Réponse à la crise des entreprises endommagées à Kamaishi), in GendA Yūji, lıDA Takashi 飯田高 \& Nakamura Naofumi (dir.), Kiki taiō no shakai kagaku 危機対応の社会 科学 (Sciences sociales de la réponse aux crises), Tōdai shaken, Tokyo daigaku shuppankai.

\section{Shin Nippon seitetsu Kamaishi} seitetsujo 新日本製鐵釜石製鉄所 (aciérie Nippon Steel Kamaishi) 2014 « Kamaishi to tomoni ikiru seitetsujo to shite, chiiki shien to jigyō no fukkyū ni torikumu » 釜石と共に生きる製鉄所 として、地域支援と事業の復旧に取り組む (L'implication du soutien régional et du rétablissement des entreprises en tant qu'aciérie à Kamaishi), in Genda Yūji \& Nakamura Naofumi (dir.), "Mochiba » no kibōgaku, Tōdai shaken, Tokyo daigaku shuppankai : 374-390.

Tōkyō daigaku shakai kagaku kenkyūjo 東京大学社会科学研究所 (Institut des sciences sociales de l'université de Tokyo) 2013

Shinsai no kioku ōraru hisutorì dainiji kōhon 震災の記憶オーラル・ヒストリー 第二次稿本 (Histoire orale du souvenir du séisme. Deuxième manuscrit), Tōkyō daigaku shakai kagaku kenkyūjo kibōgaku purojekuto 東京大学社会科学研究 所希望学プロジェクト (projet d'études sur l'espoir de l'Institut des sciences sociales de l'université de Tokyo).

\section{Uno Shigeki 宇野重規 2009}

"Kamaishi shichō to shite no Suzuki Tōmin. Chiiki no fukushi seiji to rōkaru aidentiti » 釜石市長としての鈴木東民 地域
の福祉政治とローカル・アイデンティティ (Suzuki Tōmin en maire de Kamaishi. Politique sociale régionale et identité locale), in Genda Yūji \& Nakamura Naofumi (dir.), Kibōgaku 2. Kibō no saisei, Tōdai shaken, Tokyo daigaku shuppankai : 109-136. 